\title{
Le lait vecteur des nucléides radioactifs. Prévision des conséquences d'une pollution accidentelle des pâturages (1)
}

\author{
par \\ G. MICHON
}

Le développement de l'utilisation de l'énergie nucléaire, s'il présente l'avantage non négligeable de mettre à la disposition de l'humanité une source nouvelle d'énergie s'accompagne également de risques, dont le plus préoccupant est celui de la pollution radioactive de notre environnement.

Le début fracassant de l'ère nucléaire a centré les feux de l'actualité sur les risques énormes découlant de certains emplois de cette forme de l'énergie. Il en résulte un contexte psychologique défavorable qui force les spécialistes à analyser en détail les risques réels. Il est né, de cette contrainte bénéfique, des disciplines scientifiques nouvelles telles que l'hygiène atomique et la radio-écologie qui scrutent avec attention les risques résultant pour l'homme des pollutions continues ou accidentelles de son milieu et de son alimentation.

Le lait, par son importance dans l'alimentation surtout du jeune mais également par la place qu'il occupe dans les chaînes alimentaires, est un vecteur essentiel des nucléides radioactifs tant du point de vue quantitatif que de celui de la rapidité avec laquelle il transfère la pollution depuis le milieu jusqu'à l'homme.

Utilisant les remarquables possibilités offertes par ces nucléides dans les recherches physiologiques et écologiques, les chercheurs ont pu aboutir à des résultats quantitatifs dont on ne connaît pas l'équivalent pour bien d'autres nuisances. Il est maintenant possible de tenter, dès que la pollution se produit, une estimation de ses conséquences. Ces méthodes utilisent à la fois les résultats obtenus par les physiologistes de la sécrétion lactée et les résultats des surveillances assidues des pollutions radioactives mises en place dans la plupart des pays du monde.

(1) Entretiens de Bichat 1968. Economie et Médecine animale, 1968 n 6, 342. 


\section{Données métaboliques fondamentales}

La relative aisance avec laquelle on peut détecter la présence d'un radionucléide dans un milieu biologique, mesurer sa concentration, permet de connaître de manière précise son métabolisme et notamment, après administration unique ou répétée d'établir les cinétiques des accumulations dans certains organes et les cinétiques des éliminations. La sécrétion mammaire qui nous intéresse ici peut en effet être considérée comme une excrétion et il est tout à fait aisé, théoriquement du moins, d'établir les variations de concentration d'un nucléide dans le lait en fonction du temps.

Ces données peuvent être obtenues expérimentalement, en administrant par voie orale et sous une forme aussi proche que possible de celles que l'on rencontre dans la nature, le radio-élément étudié.

Chaque traite est soigneusement pesée, la concentration en nucléide du lait mesurée, la quantité totale excrétée déterminée. Malheureusement de telles expériences, excessivement simples dans leur principe, nécessitent des moyens matériels importants et restent à l'heure actuelle peu nombreuses. Les résultats obtenus sont des moyennes obtenues sur un petit nombre d'individus, exceptionnellement une vingtaine et quelques races bovines seulement ont été étudiées. Cependant bien que la valeur statistique des moyennes obtenues soit discutable, les résultats publiés par divers auteurs sont cohérents et peuvent servir de bases sérieuses.

D'une manière générale l'évolution de la concentration d'un nucléide dans le lait, après une administration orale unique peut se mettre sous la forme d'une somme de fonctions exponentielles du temps. Le nombre des termes exponentiels de cette évolution est fonction de la complexité du métabolisme du corps étudié. Cependant dans la plupart des cas, si l'on néglige l'élimination des fractions ultimes et le plus souvent négligeables sur le plan hygiénique, la relation concentration en fonction du temps se réduit à trois ou quatre termes exponentiels.

Dans le tableau 1, figurent quelques-unes de ces relations publiées par divers auteurs. Nous nous sommes limités aux trois éléments qui présentent les plus grands risques potentiels, à savoir l'iode, le strontium, le caesium.

Toutes ces relations font apparaître que les trois nucléides sont présents très rapidement dans le lait, pratiquement dès la première traite.

Pour l'iode le maximum est atteint 12 heures après l'ingestion, pour le strontium au bout d'environ 30 heures (4) et pour le caesium au bout de 24 heures. Les concentrations correspondantes à ces maximums sont de 0,3 à 0,5 p. 100 de la quantité d'iode ingérée, 0,08 à 0,09 p. 100 de la quantité de strontium ingérée, 0,3 p. 100 de la quantité de caesium ingérée. 
TABLEAU No 1

Concentration d'un nucléide dans le lait en fonction du temps à la suite d'une ingestion orale unique

\begin{tabular}{|c|c|c|}
\hline Nucléides & & Réf. \\
\hline I & $\begin{array}{l}\mathrm{Ct}=5,6 \cdot 10-3 \cdot \mathrm{e}^{-0,94 \mathrm{t}}+7,5 \cdot 10-5 \cdot \mathrm{e}^{-0,032 \mathrm{t}}-5,7 \cdot 10-3 \cdot \mathrm{e}^{-6,63 \mathrm{t}} \\
\mathrm{Ct}=5,4 \cdot 10-3 \cdot \mathrm{e}^{-0,76 t}+0,37 \cdot 10-5 \cdot \mathrm{e}^{-0,19 t}\end{array}$ & $\begin{array}{l}1 \\
2\end{array}$ \\
\hline $\mathrm{Sr}$ & $\mathrm{Ct}=5,5 \cdot 10-4 \cdot \mathrm{e}^{-0,48 \mathrm{t}}+1,0 \cdot 10-6 \cdot \mathrm{e}^{-0,017 \mathrm{t}}-5,5 \cdot 10-4 \cdot \mathrm{e}-1,58 \mathrm{t}$ & 1 \\
\hline Cs & $\begin{array}{l}\mathrm{Ct}=3,6 \cdot 10-3 \cdot \mathrm{e}^{-0,69 t}+1,5 \cdot 10-3 \cdot \mathrm{e}^{-0,17 t}+4,0 \cdot 10-5 \cdot \mathrm{e}^{-0,023 t}-5,1 \cdot 10^{-3} \cdot \mathrm{e}^{-1,84 t} \\
\mathrm{Ct}=3,6 \cdot 10-3 \cdot \mathrm{e}^{-5 t}-4,7 \cdot 10^{-2} \cdot \mathrm{e}^{-1,23 t}+4,1 \cdot 10^{-2} \cdot \mathrm{e}^{-0,97 t}+1,65 \cdot 10^{-3} \cdot \mathrm{e}^{-0,19 t}+9 \cdot 10-5 \cdot \mathrm{e}^{-0,02 t}\end{array}$ & $\begin{array}{l}1 \\
3\end{array}$ \\
\hline
\end{tabular}


Les concentrations décroissent ensuite plus ou moins vite en fonction des périodes biologiques des éléments considérés au niveau des organes intéressés par le métabolisme en cause et éventuellement de la période physique du radionucléide.

Si l'ingestion, au lieu d'être unique, se répète régulièrement pendant un certain temps, les différents compartiments métaboliques vont se mettre en équilibre. La concentration du nucléide va croître d'une traite à la suivante pour tendre vers un plateau qui apparaîtra plus ou moins tardivement.

Pour l'iode le plateau apparaît vers le $5^{\text {me }}$ jour (5) et la quantité secrétée par litre de lait correspond à 0,5 à 1 p. 100 de l'ingestion quotidienne.

Pour le caesium l'équilibre n'est pas atteint au bout de 30 jours, la quantité secrétée par litre de lait tend vers une valeur correspondant à 1,5 p. 100 de la quantité quotidiennement ingérée (3).

Pour le strontium, dont le métabolisme met en jeu des compartiments ayant des périodes très longues, l'équilibre biologique n'est jamais réellement atteint. Cependant la plus grande part de l'augmentation est acquise dans les 15 premiers jours et les compétitions qui existent entre $\mathrm{Ca}$ et $\mathrm{Sr}$ au niveau de diverses membranes jouent un rôle plus important que la mise en équilibre. On peut, sans risque d'erreurs importantes adopter les valeurs publiées par Comar (6). Avec un régime pauvre en Ca (teneur de 0,25 p. 100) on retrouve par litre de lait 0,14 p. 100 de la quantité quotidiennement ingérée. Cette valeur tombe à 0,08 p. 100 pour un régime ayant une teneur moyenne en $\mathrm{Ca}(0,5 \mathrm{p}, 100)$ et à $0,04 \mathrm{p} .100$ si la diète est riche en $\mathrm{Ca}(1,7 \mathrm{p} .100)$.

Les résultats expérimentaux obtenus par les expériences d'administrations orales répétées sont en très bon accord avec les valeurs que l'on peut calculer par sommation à partir des fonctions obtenues à la suite d'une ingestion unique.

D'une manière générale l'évolution de la concentration résultant d'administrations répétées peut être représentée par une fonction plus simple que celles figurant au tableau 1 de la forme (7).

$\mathrm{Ct}=\mathrm{c} \mathrm{e}-\mathrm{k}_{1} \mathrm{t}\left(1-\mathrm{e}-\mathrm{k}_{2} \mathrm{t}\right)(\mathrm{A})$

ou $t$ est le temps écoulé depuis le début des administrations

$\mathrm{C}$ - $\mathrm{k}_{1}$ et $\mathrm{k}_{2}$ sont des constantes physiologiques variables avec l'élément chimique considéré.

Si l'administration cesse la concentration du nucléide dans le lait décroît en suivant une fonction sinon identique du moins très voisine de celle décrivant la partie décroissante de l'évolution observée après administration orale unique.

La figure $n^{\circ} 1$ reprise de Lengemann (3) illustre pour le Cs l'ensemble de ces évolutions.

Pour indispensables qu'elles soient ces données ne sont cependant pas immédiatement exploitables dans les circonstances pratiques 
que l'hygiéniste peut rencontrer. En effet il nous faut remonter plus haut dans la chaîne alimentaire car en pratique c'est au travers de la pollution de leur nourriture que les bovins ingéreront des nucléi-

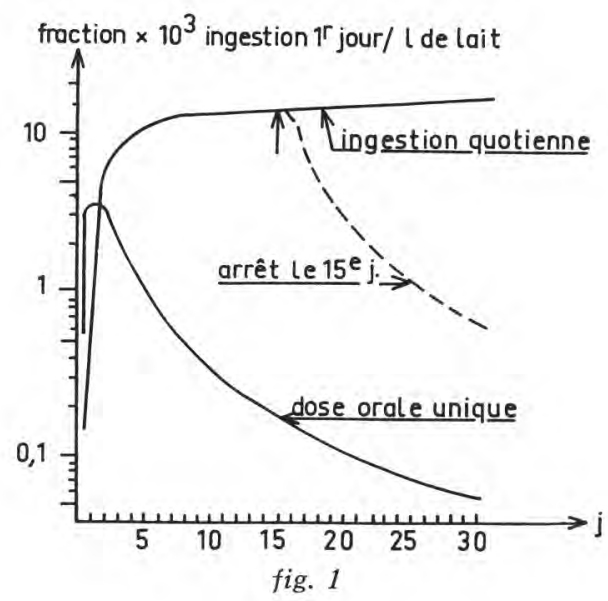

des radioactifs. L'assimilation par la voie respiratoire peut en effet être négligée dans la quasi-totalité des cas (8).

\section{Contamination d'un pâturage par un apport unique de radioéléments}

Parmi les diverses possibilités nous étudierons une situation susceptible de se produire en temps de paix. Il s'agit de l'hypothèse d'un accident survenant à un réacteur nucléaire avec libération de nucléides radioactifs. Ces nucléides libérés dans l'air vont venir polluer le sol et les végétaux et le problème à résoudre, dans le cas des pâturages sera d'apprécier les conséquences de cette pollution. Une situation analogue peut se rencontrer dans le cas d'une pluie fortement radioactive consécutive à une explosion nucléaire ayant eu lieu dans la basse troposphère.

Dans les circonstances que nous venons d'évoquer quatre nucléides radioactifs sont plus particulièrement intéressants car ils seront présents en quantités proportionnellement importantes et qu'ils sont parfaitement métabolisables par les animaux ou l'homme.

Ce sont : 1'131I de période 8 jours, le $137 \mathrm{Cs}$ et le $90 \mathrm{Sr}$ de période 30 ans, le $89 \mathrm{Sr}$ de période 65 jours (9-10).

a) Evolution de la concentration des nucléides dans le lait

Deux phénomènes nouveaux vont venir modifier le schéma précédemment établi. En effet les bovins en pâture sur les prés contaminés ne vont pas ingérer tous les jours la même quantité de 
nucléides déposés sur l'herbe du fait, d'une part de la décroissance physique des radioéléments et d'autre part d'une diminution de la quantité de nucléides fixée par les végétaux. La décroissance radioactive n'est à prendre en considération que pour l'iode 131 dont la période est relativement courte.

La perte de radioactivité par le pâturage provient du lessivage par la pluie et de la pousse de l'herbe qui par un apport d'aliment non contaminé contribue à faire diminuer la radioactivité rapportée à la masse d'aliments ingérée.

Les résultats obtenus par de nombreux auteurs à proximité des champs de tir nucléaire ou après quelques incidents survenus à des réacteurs nucléaires (8-11-12-13) montrent que cette diminution a également une allure exponentielle et qu'en moyenne on observe une diminution de moitié de l'activité tous les 15 jours si, bien sûr, la période physique du radioélément n'intervient pas.

Il en résulte que l'équation " $\mathrm{A}$ » ne représente plus l'évolution de la concentration du nucléide dans le lait rapportée à la quantité ingérée si les bovins restent sur la même pâture. La fonction prend la forme ci-après (14):

$$
C t=c e-k_{1} t\left(1-e-k_{2} t\right)\left(e-k_{3} t\right)\left(e-k_{4} t\right)(B)
$$

ou $\mathrm{k}_{3}$ représente constante de perte par la végétation

$\mathrm{k}_{4}$ est la constante radioactive de l'élément considéré.

Le tableau 2 donne des valeurs publiées pour ces différents facteurs et pour les nucléides envisagés.

TABLEAU $N^{\circ} 2$

Concentration d'un nucléide dans le lait en fonction du temps (Animaux restant en pâture sur un pré contaminé)

\begin{tabular}{|c|c|c|c|c|}
\hline & ${ }^{131} \mathrm{I}$ & ${ }^{89} \mathrm{Sr}$ & ${ }^{90} \mathrm{Sr}$ & ${ }^{137} \mathrm{Cs}$ \\
\hline \multicolumn{5}{|l|}{$\mathrm{nCi} / 1$ lait } \\
\hline $\begin{array}{cc}\mathrm{nCi} \text { ing. le } & 1^{\mathrm{er}} \text { jour } \\
\mathbf{k}_{1} & \mathbf{j}^{-1} \\
\mathbf{k}_{2} & \mathbf{j}^{-1} \\
\mathbf{k}_{y_{3}} & \mathbf{j}^{-1} \\
\mathbf{k}_{4} & \mathbf{j}^{-1}\end{array}$ & $\begin{array}{l}0,02 \\
0,29 \\
0,86 \\
0,05\end{array}$ & $\begin{array}{l}0,017 \\
0,45 \\
0,013 \\
0,05\end{array}$ & \begin{tabular}{|c|}
0,017 \\
0,45 \\
négligeable \\
0,05
\end{tabular} & $\begin{array}{l}7,310-{ }^{5} \mathrm{t}^{*} \\
0,3 \\
\text { négligeable } \\
0,05\end{array}$ \\
\hline$T \max j$ & 4 & 6 & 6 & 7 \\
\hline $\begin{array}{l}\text { Réduction de } \mathrm{Ct} \text { max d'un facteur } 10 \\
\text { en jours }\end{array}$ & 23 & 51 & 76 & 64 \\
\hline $\begin{array}{l}\text { Réduction de Ct max d'un facteur } 100 \\
\text { en jours }\end{array}$ & 43 & 106 & 154 & 133 \\
\hline Ingestion totale prévisible & $15 \times \underset{(16)}{\mathrm{Cm}}$ & $28 \times \mathrm{Cm}$ & $37 \times \mathrm{Cm}$ & $40 \times \mathrm{Cm}_{(3)}$ \\
\hline \multicolumn{5}{|c|}{ * Pour le Cs l'équation la meilleure à la forme $\mathrm{Ct}=(0,0138+\underset{(1-\mathrm{e}-\mathrm{krt}) \ldots \ldots}{0 . \ldots}$} \\
\hline
\end{tabular}


Cette fonction présente un maximum également indiqué dans le tableau. Afin de concrétiser le phénomème figurent également les temps nécessaires pour que les concentrations tombent au $1 / 10^{\mathrm{e}}$ et au $1 / 100^{\mathrm{e}}$ de la valeur maximale.

On peut constater que le lait restera pollué longtemps puisque pour le $\mathrm{Cs}$ ce n'est que 76 jours après la pollution que la concentration tombe au dixième de la valeur maximale observée le $6^{\text {me }}$ jour. La figure 2 représente pour l'131I cette évolution.

La connaissance de ces fonctions permet cependant plus que la prévision des concentrations futures en agent polluant dans le lait, elle permet également de prévoir la quantité de nucléides qui véhiculée par le lait, vecteur prépondérant dans ce type d'accidents, sera ingérée par un individu moyen. Seule cette donnée permet d'apprécier les conséquences pour l'homme de la pollution et de choisir éventuellement les actions à entreprendre pour y remédier.

b) Détermination de la quantité ingérée du fait d'une pollution unique

L'intégration des précédentes fonctions entre le temps zéro et l'infini représente à un facteur près la quantité de radioéléments qui serait ingérée par l'homme consommant le lait produit par les vaches restant sur la pâture contaminée.

Pour obtenir cette ingestion intégrée il faut connaître :

- le volume de lait consommé par l'individu moyen,

- le temps qui s'écoule entre la production du lait et sa consommation,

- l'ingestion journalière initiale de nucléides par une vache.

La première de ces données est facile à obtenir et l'on peut simplifier en prenant par exemple une consommation moyenne journalière de 11 .

La seconde est fonction des circuits de distribution. La connaissance de ce délai de consommation est importante pour les nucléides à périodes assez courtes pour décroître de manière substantielle entre le moment de la production et celui de la consommation. Dans le cas de l'iode 131 et pour un délai de 3 jours la concentration ne sera plus à la consommation que les $77 \mathrm{p}$. 100 de la concentration à la production.

La troisième donnée est la plus difficile à obtenir et dans la plupart des cas elle ne peut être estimée qu'avec une marge trop grande d'incertitude pour être utilisable.

Il est cependant possible de tourner cette difficulté. En normalisant la fonction pour une ingestion initiale de $1 \mu \mathrm{Ci}$ on peut à la fois calculer l'aire de la courbe et calculer la concentration au moment du maximum. On obtient ainsi un rapport qui reste valable quelle que soit l'ingestion initiale par la vache. L'ingestion intégrée pour 
l'homme est alors égale au produit de ce facteur par la concentration dans le lait au moment du maximum.

Ces valeurs figurent au bas du tableau 2. Par exemple si la concentration maximale en $90 \mathrm{Sr}$ est de $10 \mathrm{mCi}$ par litre de lait l'ingestion totale projetée sera de $37 \times 10=370 \mathrm{mCi}$.

Il faut cependant connaître la concentration maximale c'est-àdire faire plusieurs mesures et attendre quelques jours avant de pouvoir faire l'estimation et il s'agit-là d'une perte de temps dont on verra l'importance au paragraphe suivant.

Le même procédé peut être utilisé en calculant le même rapport :

$$
\mathrm{F}=\frac{\text { Aire totale de la fonction de } 0 \text { à } \infty}{\text { Concentration dans le lait }}
$$

pour chaque jour qui suit l'événement contaminant.

Pour calculer l'ingestion totale par l'homme consécutive à cet événement il suffit alors de connaître la date de l'événement, la date de production d'un échantillon et sa teneur en nucléides rapportée

TABLEAU N० 3

Valeur du coefficient « $F$ » permettant de calculer l'ingestion totale

\begin{tabular}{c|c|c}
\hline $\begin{array}{c}\text { Jours après la contamination } \\
\text { initiale des pâturages }\end{array}$ & ${ }^{131} \mathrm{I}$ & \\
\hline 1 & ${ }^{137} \mathrm{Cs}$ \\
2 & 31,7 & \\
3 & 19,4 & 215 \\
4 & 16,2 & 77 \\
5 & 15,1 & 54 \\
6 & 14,9 & 46 \\
7 & 15,4 & 42 \\
8 & $17^{*}$ & 41 \\
9 & 17,3 & 40 \\
10 & $19^{*}$ & 40 \\
12 & 20,4 & 40 \\
14 & & 41 \\
20 & 59 & 42 \\
& & 45 \\
\end{tabular}

* Valeurs obtenues par extrapolation graphique.

à cette date. On peut alors sélectionner le rapport $\mathrm{F}$ adéquat, le multiplier par la concentration trouvée et le volume moyen de lait consommé pour connaître l'ingestion totale par l'homme. Les valeurs $\mathrm{F}$ ainsi calculées figurent au tableau 3. Elles ne tiennent pas compte de la décroissance du nucléide entre le moment de la traite et le moment de la consommation.

Pour l'iode 131 il y a lieu de tenir compte de ce délai et de corriger ces valeurs en les multipliant par $F^{\prime}=e-0,86 t^{\prime}$ où $t^{\prime}$ est le nombre de jours s'écoulant entre production et consommation. 
Ces valeurs $\mathrm{F}$ permettent, dès que l'on a connaissance de la pollution et éventuellement dès le premier jour, d'évaluer sur le plan de l'ingestion par l'homme la quantité totale qui serait ingérée en l'absence de toutes mesures d'ordre sanitaire. Il s'agit-là d'une méthode très pratique basée sur des données moyennes valables sans risques d'erreurs considérables dans de nombreuses circonstances.

Par exemple si l'on observe le $2^{\text {me }}$ jour après la contamination une teneur de $60 \mu \mathrm{Ci}$ d'131I par litre lait, un enfant consommant 1 litre de lait par jour absorbera au total :

$19,2 \times 60=1152 \mu \mathrm{Ci}$ d'131I

s'il consomme le lait produit par la ferme. S'il s'écoule un délai de 3 jours entre production et consommation il ingérera :

$$
1152 \times 0,77=887 \mu \mathrm{Ci} \mathrm{d}^{\prime} 131 \mathrm{I}
$$

Dans certaines situations il est possible de raffiner et d'obtenir un modèle plus précis.

Dans le cas des réacteurs nucléaires en effet la zone qui serait contaminée par un accident peut être délimitée et étudiée. On peut alors établir un rapport entre la contamination du lait dans cette zone et la quantité de nucléides déposée par $\mathrm{m}^{2}$ de sol.

L'établissement de ce rapport suppose que l'on connaisse :

a) Le pourcentage de l'activité déposée effectivement retenu par les végétaux. Ce pourcentage varie avec la nature de la couverture végétale (8).

b) La supercie moyenne broutée par chaque vache en un jour en formulant l'hypothèse que la totalité du dépôt radioactif se trouve fixée sur les parties des végétaux effectivement broutées ou bien en estimant le rapport adéquat.

Par prospection aérienne il est possible de dresser rapidement une carte des activités déposées au sol exprimées en curie ou sousmultiple par unité de surface. Dans une région où l'auto-consommation est forte l'hygiéniste pourra interdire la consommation locale si besoin est, alors que la méthode précédente qui ne se base que sur l'analyse que de quelques échantillons de laits de grands mélanges néglige les risques très localisés mais beaucoup plus importants.

Une tentative de cette nature a été faite par Garner (17) et le tableau 4 résume l'ensemble des résultats obtenus. Il est aisé à partir de ces résultats d'utiliser la méthode de prévision déjà décrite.

Il est même possible d'aller plus loin dans le domaine de la prévision et d'estimer directement les conséquences d'un accident type survenant à un réacteur connu. Ces estimations qui ne sont valables que dans des cas spécifiques reposent sur la connaissance ou la détermination de paramètres physiques tels que la dispersion des particules radioactives émises par une cheminée en fonction des 
$T A B L E A U N^{\circ} 4$

Activité estimée du lait (en $\mu \mathrm{Ci} / \mathrm{l}$ ) à la suite d'un dépôt initial de $1 \mu \mathrm{Ci} / \mathrm{m}^{2}$

\begin{tabular}{|c|c|c|c|c|c|c|c|}
\hline $\begin{array}{l}\text { Jours après } \\
\text { le dépôt }\end{array}$ & ${ }^{131} \mathrm{I}$ & ${ }^{133} \mathrm{I}$ & ${ }^{132} \mathrm{Te}$ & ${ }^{90} \mathrm{Sr}$ & ${ }^{59} \mathrm{Sr}$ & ${ }^{140} \mathrm{Ba}$ & ${ }^{137} \mathrm{Cs}$ \\
\hline 1 & 0,12 & 0,060 & 0,0029 & 0,006 & 0,006 & 0,0028 & 0,01 \\
\hline 2 & 0,16 & 0,038 & 0,0072 & 0,015 & 0,014 & 0,0058 & 0,06 \\
\hline 3 & 0,16 & 0,019 & 0,0090 & 0,021 & 0,020 & 0,0075 & 0,14 \\
\hline 4 & 0,15 & 0,009 & 0,0090 & 0,025 & 0,023 & 0,0085 & 0,21 \\
\hline 5 & 0,15 & 0,004 & 0,0082 & 0,027 & 0,025 & 0,0090 & 0,26 \\
\hline 6 & 0,14 & 0,002 & 0,0071 & 0,028 & 0,026 & 0,0092 & 0,29 \\
\hline 7 & 0,13 & 0,001 & 0,0059 & 0,029 & 0,027 & 0,0092 & 0,32 \\
\hline 8 & 0,12 & & 0,0049 & 0,030 & 0,027 & 0,0091 & 0,35 \\
\hline 9 & 0,11 & & 0,0040 & 0,030 & 0,027 & 0,0089 & 0,36 \\
\hline 10 & 0,10 & & 0,0033 & 0,030 & 0,027 & 0,0086 & 0,39 \\
\hline 21 & 0,04 & & & 0,031 & 0,023 & 0,0049 & 0,48 \\
\hline
\end{tabular}

Hypothèses: a) 25 p. 100 du dépôt est retenu par les parties comestibles des végétaux.

b) La décroissance d'activité de l'herbe est uniquement due à la décroissance radioactive.

c) Les animaux sont en pâture libre et ne reçoivent aucune alimentation complémentaire.

d) Les animaux consomment environ $9 \mathrm{~kg}$ de matière sèche par jour sur un pâturage produisant $550 \mathrm{~kg}$ de matière sèche par hectare. 
conditions météorologiques et des paramètres qui régissent la rétention par les végétaux des particules ou des gaz radioactifs. L'étude de ces facteurs sort du cadre biologique de cet exposé. Le lecteur intéressé pourra se reporter à quelques travaux cités en bibliographie (12-18-19).

c) Evaluation de l'intérêt de quelques mesures simples d'ordre sanitaire :

Si l'évaluation faite au paragraphe précédent de la quantité totale de nucléides ingérée par l'homme en l'absence de toute mesure conduit à un équivalent de dose supérieur à certaines limites acceptables dont il ne sera pas discuté ici, l'expert sanitaire responsable est amené à entreprendre des actions visant à réduire autant que faire se peut l'ingestion des nucléides par l'homme.

Les premières mesures qui viennent à l'esprit consistent :

a) A faire cesser la consommation de lait contaminé par l'homme.

b) A nourrir les bovins avec une nourriture exempte de contamination.

TABLEAU N 5

Efficacité de l'élimination du lait contaminé de l'alimentation humaine

\begin{tabular}{|c|c|c|c|}
\hline \multirow{2}{*}{$\begin{array}{l}\text { Ingestion réelle } \\
\text { en p. } 100 \text { de l'ingestion } \\
\text { totale prévue }\end{array}$} & \multicolumn{3}{|c|}{$\begin{array}{l}\text { Nombre de jours au bout duquel la } \\
\text { consommation de lait contaminé doit cesser }\end{array}$} \\
\hline & ${ }^{131} \mathbf{I}$ & ${ }^{137} \mathrm{Cs}$ & (3) \\
\hline 10 p. 100 & 2 & 6 & \\
\hline 25 p. 100 & 5 & 12 & \\
\hline 50 p. 100 & 8 & 24 & \\
\hline
\end{tabular}

TABLEAU $N^{\circ} 6$

Efficacité de l'élimination du fourrage contaminé de l'alimentation des bovins

\begin{tabular}{|c|c|c|c|c|}
\hline \multirow{2}{*}{$\begin{array}{l}\text { Ingestion réelle par } \\
\text { l'homme en p. } 100 \text { de } \\
\text { l'ingestion totale } \\
\text { prévue }\end{array}$} & \multicolumn{4}{|c|}{$\begin{array}{l}\text { Nombre de jours au bout duquel la } \\
\text { consommation de fourrage contaminé } \\
\text { doit cesser }\end{array}$} \\
\hline & ${ }^{{ }^{131} \mathbf{I}}$ & \begin{tabular}{|l|}
${ }^{137} \mathrm{Cs}$ \\
\end{tabular} & ${ }^{90} \mathrm{Sr} \quad$ (15) & ${ }^{89} \mathrm{Sr}$ \\
\hline 10 p. 100 & 1 & 2 & 2 & 2 \\
\hline 25 p. 100 & 3 & 4 & 7 & 6 \\
\hline 50 p. 100 & 6 & 15 & 13 & 17 \\
\hline
\end{tabular}


Nous allons envisager successivement ces deux situations.

Ainsi que nous l'avons dit précédemment l'aire de la courbe donnant la concentration dans le lait est proportionnelle à la quantité de nucléides ingérée, du fait de la consommation du lait, par l'homme entre le temps 0 et l'infini. Si à un jour donné " $t$ " l'ingestion de lait pollué cesse, l'ingestion résultante est proportionnelle à l'aire comprise entre 0 et " $t$ ». Cette aire est représentée en hachures sur la figure 2 . On voit que plus la décision est prise tôt plus l'ingestion réelle sera petite par rapport à l'ingestion totale prévue, d'où l'intérêt qu'il $\mathrm{y}$ a à pouvoir faire une estimation précoce de cette dernière quantité.

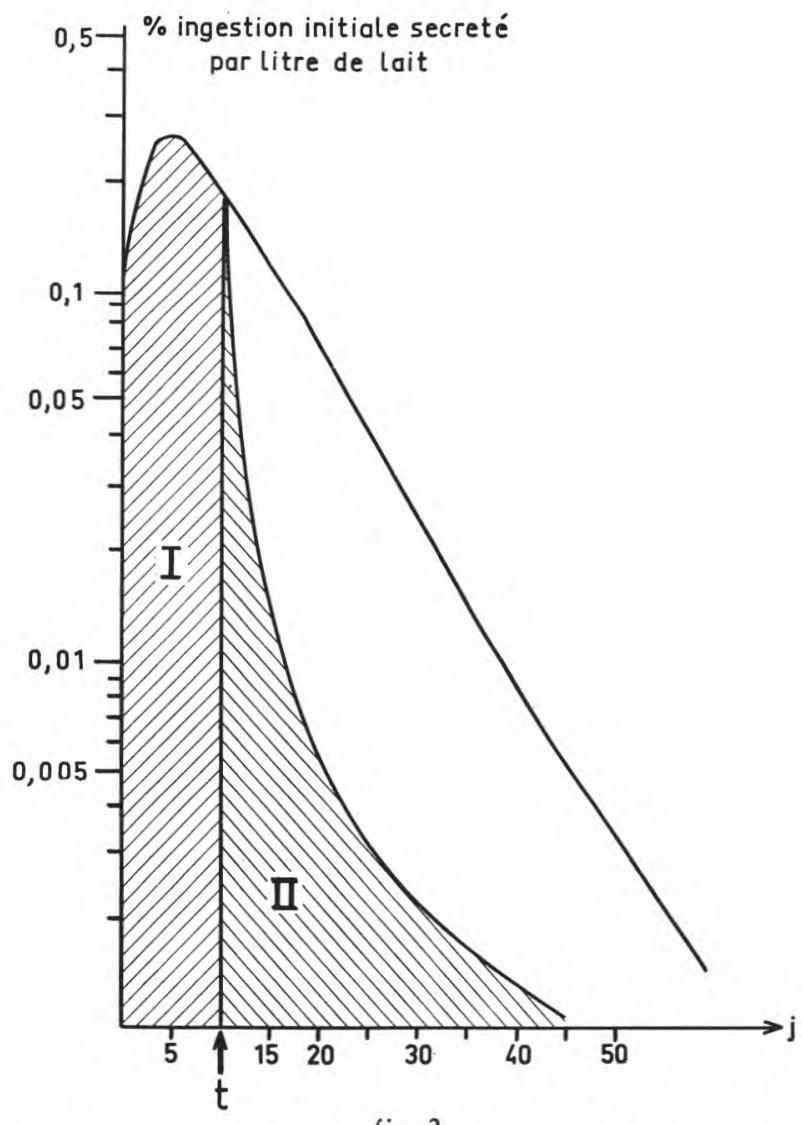

fig. 2

I aire proportionnelle à l'ingestion par l'homme si au jour « $t$ » le lait est éliminé.

II + I idem si le fourrage est éliminé.

Le tableau 5 donne quelques résultats des réductions qui sont obtenues en éliminant le lait contaminé de l'alimentation. On peut voir qu'en éliminant le lait contaminé par du 137Cs, 6 jours après le 
début de la consommation on évite l'ingestion de 90 p. 100 de la quantité initialement prévue.

Si par contre la nourriture des vaches est modifiée au temps " $t$ » et devient exempte de radioéléments le lait restera contaminé pendant un certain temps. L'ingestion totale pour un individu continuant à consommer le lait produit par ce troupeau sera la somme de l'aire comprise entre le temps 0 et le temps «t» auquel il faut ajouter l'aire de la fonction d'élimination par le lait comprise entre le temps du maximum et l'infini. Cette sommation est également représentée sur la figure 2 .

Le tableau 6 donne quelques résultats ainsi obtenus. En comparant les valeurs figurant dans les tableaux 5 et 6 on constate que pour obtenir le même facteur de réduction les mesures visant à modifier l'alimentation du cheptel doivent être prises plutôt que celles concernant l'alimentation humaine. Il peut a priori sembler plus efficace d'interdire la consommation du lait contaminé. Cepen-

\section{TABLEAU N $N^{\circ} 7$}

Comparaison entre l'efficacité des mesures d'ordre sanitaire

\begin{tabular}{|c|c|c|c|c|}
\hline \multirow[t]{2}{*}{$\begin{array}{l}\text { Type de mesure } \\
\text { prise }\end{array}$} & \multicolumn{4}{|c|}{$\begin{array}{l}\text { Ingestion totale par l'homme } \\
\text { exprimée en multiple de la concentration } \\
\text { maximale et pour une consommation de } \\
1 \text { litre par jour }\end{array}$} \\
\hline & ${ }^{131} \mathrm{I}$ & ${ }^{137} \mathrm{Cs}$ & ${ }^{90} \mathrm{Sr}$ & ${ }^{89} \mathrm{Sr}$ \\
\hline Aucune & $15 \mathrm{Cm}$ & $40 \mathrm{Cm}$ & $37 \mathrm{Cm}$ & $28 \mathrm{Cm}$ \\
\hline $\begin{array}{l}\text { Interruption de la } \\
\text { consommation du lait* }\end{array}$ & $3 \mathrm{Cm}$ & $62 \mathrm{Cm}$ & $6 \mathrm{Cm}$ & $5 \mathrm{Cm}$ \\
\hline $\begin{array}{l}\text { Interruption de la } \\
\text { consommation du fourrage* }\end{array}$ & $46 \mathrm{Cm}$ & $116 \mathrm{Cm}$ & $10 \mathrm{Cm}$ & $9 \mathrm{Cm}$ \\
\hline $\begin{array}{l}\text { P. } 100 \text { d'ingestion supplémentaire } \\
\text { dû à la } 2^{\circ} \text { procédure par } \\
\text { rapport à la } 1^{e}\end{array}$ & 53 p. 100 & 87 p. 100 & $66 \mathrm{p} .100$ & 80 p. 100 \\
\hline
\end{tabular}

dant une telle mesure peut ne pas être possible. De toute manière elle désorganise complètement les circuits de distribution et si l'on ne peut obtenir de lait provenant des zones exemptes de pollution les conséquences peuvent être graves. Il convient d'apprécier soigneusement les inconvénients et de les confronter avec ceux qui résulteraient de l'apport supplémentaire de nucléides dû au fait que l'action au lieu d'être entreprise au niveau de l'alimentation humaine 
l'est au niveau zootechnique. Tout n'est fonction que des circonstances et surtout du moment où la mesure peut effectivement être prise.

A titre d'exemple, le tableau 7 compare les ingestions prévues, les mesures sont prises au moment où la concentration du nucléide atteint son maximum. On peut constater qu'à ce moment l'augmentation de l'ingestion varie suivant les nucléides de 50 à 90 p. 100. Ces valeurs sont importantes mais peuvent être trompeuses. En fait le choix ne doit pas être fait en fonction de ces données exprimées en pourcentages mais sur les valeurs absolues puisque l'on peut admettre l'ingestion d'une certaine quantité de nucléides sans qu'il en résulte aucun inconvénient grave pour les consommateurs.

Si l'ingestion reste faible un doublement de la dose résultante peut être de conséquence négligeable. Si par contre la prévision montre que l'on risque de dépasser les quantités considérées comme pouvant être dangereuses la situation est totalement différente et il convient d'agir sur l'alimentation humaine.

\section{CONCLUSIONS}

Si les résultats publiés par les différents auteurs cités sont susceptibles d'être appliqués aux situations zootechniques existant en France il est possible de prévoir avec une raisonnable précision la quantité de nucléides véhiculée par le lait, à la suite d'un incident nucléaire, ingérée par un individu de la population.

Cette donnée est la seule qui puisse permettre une évaluation de l'équivalent de dose intégré qui serait reçu par tel ou tel organe. Seule cette évaluation permet d'apprécier la nuisance potentielle de l'accident et, en fonction des risques que l'on entend faire supporter à la population, permet à l'hygiéniste responsable de prendre les mesures appropriées et d'en mesurer l'efficacité sur le plan de la protection contre les rayonnements.

\section{Conclusions}

If the results which have been published by the above - mentioned authors can be applied to the zootechnical situations existing in France, it is possible to determine with a sufficient precision the quantity of "nucleids " vehiculed by milk as a result of a nuclear accident, and ingested by a human being.

It is but the only one element which can allow an evaluation of the equivalent of integrated amount which would be received by such or such an organ. 
This evaluation only gives the possibility to appreciate the potential damages of the accident, and, according to the risks the population is supposed to be submitted, enables hygienists to take appropriate measures, and to test their values concerning the protection against radiations.

\section{$R$ é $\mathbf{s}$ u $\mathrm{m}$ é}

Le lait, vecteur des nucléides radioactifs prévision des conséquences d'une pollution accidentelle des pâturages

L'auteur dans une courte synthèse des données physiologiques et agronomiques indique comment, dans le cas d'une pollution radioactive d'un pâturage par le l'131I, du $90 \mathrm{Sr}$, du $89 \mathrm{Sr}$ ou du $137 \mathrm{Cs}$ il est possible de prévoir :

a) l'évolution de la concentration en nucléides dans le lait,

b) les quantités qui seront ingérées par un consommateur humain,

c) les mesures sanitaires à prendre et l'intérêt qu'elles présentent.

\section{S u m m a r y}

Milk, as a vector of radioactive nuclides

predicting the consequences of an accidental pollution

$$
\text { of pastures }
$$

In a short synthesis of physiological and agricultural data, the author indicates how, in the case of a radioactive pollution of a pasture by $131 \mathrm{I}, 90 \mathrm{Sr}, 89 \mathrm{Sr}$ and $137 \mathrm{Cs}$, it is possible to estimate :

1) the evolution of the nuclide concentration in milk,

2) the quantities that will be ingested by man,

3 ) the sanitary measures to be taken and their interest.

\section{B i bliographie}

[1] GARNER (R. J.) - A mathematical analysis of the transfer of fission products to cows' milk. Health Physics, 1967, 13, p. 205-212.

[2] Lengemann (F. W.). - Predicting the total projected intake of radioiodine from milk by man. II - The situation where counter measures are employed. Health Physics, 1966, 12, p. 831-835.

[3] Lengemann (F. W.), Wentworth (R. A.), Hiltz (F. L.). - Predicting the Cesium 137 intake from milk of a human population after a single, short-term deposition of the radionuclide. Health Physics, 1968, 14, p. 101-109.

[4] Souire (H. M.), Middleton (L. J.), Sansom (B. F.), Coid (C. R.) - Experiments on the Metabolism of Certain Fission Products in Dairy Cows. In Exterman : Radioisotopes in scientific research. Volume IV, p. 207 à 220, Pergamon 1958. 
[5] GaRner (R. J.), Jones (H. G.) - Fission products and the dairy cow. IV The metabolisme of ${ }^{131}$ I following single and multiple doses. J. Agric Sci., 1960 , 55, p. 387-390.

[6] Comar (C. L.), Wasserman (R. H.), Twardock (A. R.). - Secretion of calcium and strontium into milk. Health Physics, 1961, 7, p. 69-80.

[7] Lengemann (F. W.) - Predicting the total projected intake of radioiodine from milk by man. I - The situation where no counter measures are taken. Health Physics, 1966, 12, p. 885-930.

[8] BaRTH (D. S.), SEAL (M. S.). - Radioiodine transport through the ecosystem, air-forage cow-milk using a synthetic dry aerosol. In ABERG B. - Ecological Concentration Process, p. 151-158, Pergamon 1967.

[9] Michon (G.). - Organisation d'un contrôle de la pollution radioactive du lait. Cas des radioisotopes de courtes périodes. Bul. Acad. Vét., 1963, 36, p. $282-285$.

[10] Mrchon (G.). - Organisation d'un contrôle de la pollution radioactive du lait. Cas des radioisotopes de longues périodes. Bull. Acad. Vét., 1963, 36, p. $463-465$.

[11] Bustad. - Biology of radioiodine. Pergamon 1964.

[12] Chamberlain (A. C.), Chadwick. - Transport of iodine from atmosphère to ground. Rapport AERE, R 4870 , mars 1965.

[13] THOMPSON. - Effective half live of fallout radionuclide on plants with emphasis on Iodine 131. Rapport UCRL 12 388, janvier 1965.

[14] Anonyme. - Background material for the development of radiation protection standards. Protective action guides for ${ }^{89} \mathrm{Sr}{ }^{80} \mathrm{Sr}{ }^{137} \mathrm{Cs}$. Rapport fédéral Radiation Council, $\mathrm{n}^{\circ}$ 7, mars 1965.

[15] Comar (C. L.), Lengemann (F. W.). - General principles of the distribution and movement of artificial fallout through the biosphere to man. ABERG B. - Ecological Concentration Process, p. 1-18, Pergamon 1967.

[16] Lengemann (F. W.). - Predicting the total projected intake of radioiodine from milk by man. Modification of the original equation. Health Physics, $1967,13,5$, p. $521-522$.

[17] GARNER (R. J.). - An assessment of the quantities of fission products likely to be found in milk in the event of aerial contamination of agricultural land. Nature G.B., 1960, 186, 4730, p. 1063-1064.

[18] Stigall (G. E.), Leary (A. G.). - Prediction of ${ }^{131}$ I in Milk for ProtectiveAction Planning. Nuclear Safety, 1966, 3, 3, p. 364-370.

[19] BRYANT (P. M.). - Derivation of working limits for continuous release rates of ${ }^{90} \mathrm{Sr}$ and ${ }^{137} \mathrm{C} s$ to atmosphere in a milk producing area. Rapport AHSE (RP), R 60, juillet 1965 . 